preparation, disinfection and obturation. These chapters explain the nature of the problem facing the clinician, how to prevent complications, and their management in the event of occurrence.

Also covered are key areas such as perforations, root and instrument fractures and re-treatments. The book imbibes the reader with essential information on the materials and techniques required, whilst again guided by excellent clinical photography and key points.

In conclusion, this book is a must for any dental centre or specialist's library. Each chapter condenses the information into easily digested segments and key points. The text is expertly illustrated throughout with first class clinical photography and clever illustrations. A particular highlight is the way scientific findings have been incorporated and referenced throughout the text, thus bringing evidence based learning to life. As a result, this book could be strongly recommended to any dentist performing endodontic treatment.

M. Brennand Roper

\section{OXFORD HANDBOOK OF CLINICAL DENTISTRY, 5TH EDITION}

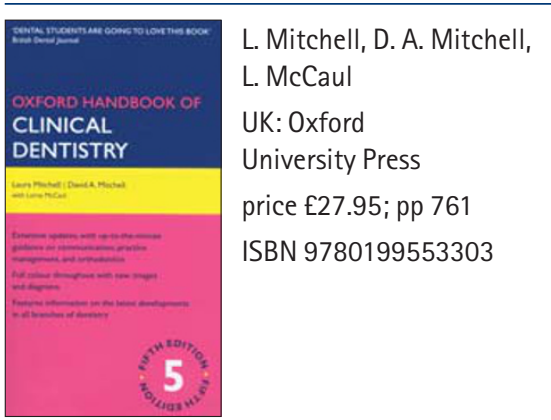

Dental students are going to love this book!

As a recent graduate, I can say that dental students love this book and have done so since the first edition. Now, as hospital trainees, this book is still in all of our bags.

Its clearly defined chapters offer excellent and concise best principles for nearly all aspects of dentistry. It is an essential aide memoire for clinical practice, whether dealing with an obscure presentation of phemphigus or the splinting of an intruded central incisor.

We all need a quick reminder or reassurance from time to time, and this book is it.
The new edition of this pocket guide covers the whole of clinical dentistry in a brief but concise manner. The main updates to this edition have been the revision of the restorative and periodontology sections, plus the inclusion of many more colour pictures and diagrams distributed throughout the text. Finally, to the delight of every student scrambling towards finals, the section on bisphosphonates has been expanded to reflect its increasing importance to the practice of modern dentistry.

\section{G. Logan}

\section{CASE REPORTS IN PEDIATRIC DENTISTRY}

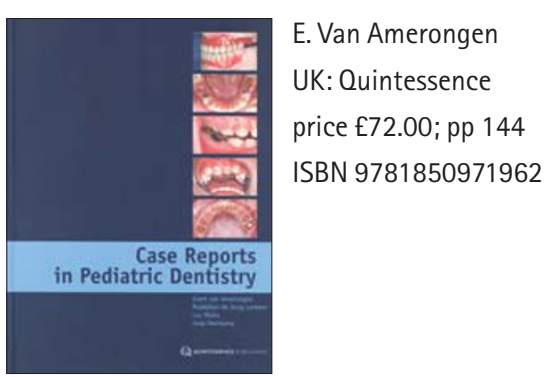

This book is a concise collection of 16 case reports related to a variety of topics in paediatric dentistry from centres throughout Europe, including the UK. The authors have taken this unique approach following the success of similar publications in the Netherlands. The aim is to 'serve as a reference for specialists in paediatric dentistry, but also help general practitioners in solving clinical problems related to their paediatric patients'.

The book is divided into 16 chapters; each one is dedicated to a clearly specified topic. These range from the commonplace to the more unusual, a feature which commends this text to generalists and specialists alike. Each report follows a structured approach, comprising of age category, definition, case history, examination, investigations, diagnosis, treatment options, follow-up/evaluation and discussion. In all cases, the rationale for the chosen treatment option is explored to give the reader a thorough understanding of the treatment aims. The length of post treatment follow-up is to be applauded in many cases. The reports are well referenced and will encourage the reader to carry out further personal research. It is interesting to learn that the approach taken in other centres may vary slightly from the UK and this may serve as a talking point among more experienced practitioners.

The layout of each chapter is appealing with concise paragraphs and key messages highlighted in separate boxes. There are over 300 colour clinical photographs which illustrate the cases superbly. These are supplemented by high quality reproductions of all radiographs.

The text itself is well written, however, there are a few instances of unusual turns of phrase. It is worth noting that the majority of these case reports are from countries where English is not the first language, and notwithstanding a few minor exceptions, the translation is excellent. There are also some minor discrepancies in the text, but these are few and far between.

Overall, the authors have fulfilled their objectives with regard to their target groups. I would go further to suggest that it would also be of interest to undergraduate and postgraduate students who would benefit from reading the relevant case following a more formal introduction to the topic. The suggestion is that this book will be followed by similar publications, and as such could form a 'case library' in dental schools and paediatric dental clinics.

S. Lucey 